\title{
Brückenhypothesen - Kritik der ökonomischen Theorie der Ziele Critique of the Economic Theory of Human Goals
}

\author{
Mateusz Stachura* \\ Ruprecht-Karls-Universität Heidelberg, Institut für Soziologie, Sandgasse 9, 69117 Heidelberg, Germany \\ E-Mail: mateusz.stachura@soziologie.uni-heidelberg.de
}

Zusammenfassung: Im vorliegenden Aufsatz wird eine institutionentheoretische Alternative zu Siegwart Lindenbergs Modell der theoriereichen Konstruktion von Brückenhypothesen entwickelt. Lindenbergs Modell richtet sich sowohl gegen theoriearme, empirische Verfahren der Konstruktion von Brückenhypothesen als auch gegen bloße Ad-hoc-Annahmen über Präferenzen sozialer Akteure. Durch die Anwendung des Nachfragegesetzes auf die Relation zwischen Präferenzen und Metapräferenzen gelingt es Lindenberg, eine innovative Theorie der Ziele zu entwickeln. In der hier aufgeführten Kritik an dieser Theorie wird jedoch gezeigt, dass Lindenberg die Ad-hoc-Annahmen nicht eliminiert, sondern nur an eine andere, weniger offensichtliche Einsatzstelle verschoben hat. Als Alternative zur ökonomisch orientierten Theorie der Ziele wird ein an Weber orientiertes Verfahren zur theoriereichen Konstruktion von Brückenhypothesen entwickelt. Durch die Kontrastierung der Informationen über die Restriktionen und die Art der Handlungsregeln erlaubt das Verfahren Rückschlüsse auf die erklärungsfähigen Ziele des Handelns.

Summary: This essay develops an institutional alternative to Siegwart Lindenberg's "theory rich bridge assumptions". Lindenbergs model speaks against "theory poor" empirical assessments in the construction of bridge assumptions as well as against using ad hoc hypotheses about preferences of social actors. By applying the law of demand to the relation between preference and meta-preference, Lindberg develops an innovative "theory of human goals". The criticism of the "theory of human goals" given here shows that Lindenberg does not eliminate ad hoc assumptions but switches these to a different position in the model. As an alternative to the economically oriented "theory of human goals" the author develops a Weberian model to construct "theory rich bridge assumptions". By contrasting the information on restrictions and the character of action-rules this model allows to draw conclusions about the explicable aims of actions.

\section{Das Problem}

Die Vertreter des Makro-Mikro-Makro-Modells der soziologischen Erklärung (MSE) sind sich einig, dass die zentrale Schwierigkeit, die mit der Modellanwendung verbunden ist, nicht die Mikro-Ebene, sondern die Makro-Mikro-Verknüpfung betrifft. ${ }^{1}$ Auf der Mikro-Ebene werden Alternativen aus einem gegebenen Alternativenraum nach einer klaren „Logik“ selektiert. Diese „Logik der Selektion“ basiert auf dem nomologischen Erklärungsgesetz, das besagt, dass aus verfügbaren Handlungsalterna-

\footnotetext{
* Für wertvolle Kommentare und weiterführende Kritik danke ich Christian Etzrodt, Thomas Schwinn, den Herausgebern und nicht zuletzt den beiden anonymen Gutachtern dieser Zeitschrift.

1 Vom Aggregationsproblem wird in der vorliegenden Abhandlung abstrahiert. Zum Modell der Makro-MikroMakro der soziologischen Erklärung allgemein vgl. Albert 2005, 2007, Coleman 1991: 7-29., Esser 1993: 91-118, Etzrodt 2007, Greshoff 2006, Greve et al. 2008).
}

tiven diejenigen ausgewählt werden, deren erwartbare Folgen im Lichte gegebener Präferenzen am höchsten bewertet werden. Wie der Alternativenraum selbst zustande kommt, wird jedoch nicht auf der Mikro-Ebene entschieden, sondern im Schritt von der Makro- auf die Mikro-Ebene. Während auf der Mikro-Ebene nur die Auskunft erteilt werden kann, dass die gewählte Alternative mit höherer Nutzenerwartung verbunden ist, muss die viel grundlegendere Frage, welcher Nutzen dabei eigentlich erwartet wird, an der „Schnittstelle“ zwischen Makro- und Mikro-Ebene beantwortet werden. Dieser erste Schritt im MSE wird als „Logik der Situation" bezeichnet.

Was diese „Logik der Situation“ genau beinhaltet, bleibt unklar, insofern das MSE kein weiteres nomologisches Gesetz außer jenem auf der MikroEbene kennt. Und von diesem „nomologischen Kern selbst geht ... so gut wie keine theoretische Steuerung für die empirische Erhebung der Nutzenargumente (Güter, Präferenzen) aus“ (Lindenberg 
1996b: 129). Mit anderen Worten: Aus der Annahme, dass Menschen mit ihren Handlungen immer den größtmöglichen Nutzen erzielen wollen, kann nicht auf die Inhalte dieser Nutzenbestrebungen geschlossen werden. Zwischen der Makro-Ebene der sozialen Situation und dem nomologischen Erklärungsgesetz auf der Mikro-Ebene tut sich eine gravierende Kluft auf.

Gravierend ist sie deshalb, weil sie den Anspruch des MSE auf die Vollständigkeit der Erklärung sozialen Handelns unterminiert. Eine solche Vollständigkeit erfordert die Rückführung der Erklärung auf diejenigen Ursachen, die zugleich subjektiv gemeinte Gründe oder Motive des Handelns sind. In Anlehnung an Max Weber könnte man von einer verstehenden und dadurch erklärenden Soziologie sprechen (Weber 1976: 1). Um den Vollständigkeitsanspruch aufrechterhalten zu können, muss nun die Kluft zwischen Situation und Selektion „überbrückt“ werden.

Das Problem der Konstruktion von Brückenhypothesen betrifft nicht nur den individualistischen Ansatz, sondern jede soziologische Erklärung, wenngleich es forschungspraktisch meistens unreflektiert übergangen wird. Im Rahmen des individualistischstrukturalistischen Ansatzes lassen sich drei Strategien identifizieren, wobei nur eine das Kriterium der theoretischen Fundiertheit erfüllt. Die erste, unbefriedigende Strategie besteht in Ad-hoc-Annahmen über die Nutzenargumente, Präferenzen oder Ziele einer Handlung. Wenn z.B. der Rückgang der Kinderzahlen in den entwickelten Industrienationen mit der Steigerung von Opportunitätskosten aufgrund verbesserter Berufschancen für Frauen erklärt wird, dann beruht dies auf der Ad-hoc-Annahme, dass die Handlungssituation der Akteure durch genau zwei Nutzenargumente bestimmt wird: Beruf auf der einen und Eigenwert des Kindes auf der anderen Seite. Solche Ad-hoc-Annahmen sind keineswegs per se inadäquat. Ihr Problem besteht jedoch darin, dass ihre explanativen Erfolge anderen, möglicherweise besseren Erklärungen den Weg versperren. Im Grenzfall verlieren die Ad-hoc-Nutzenannahmen den Bezug zum subjektiv gemeinten Sinn der Akteure, womit der Rahmen einer erklärenden und verstehenden Soziologie gesprengt würde.

Die zweite Strategie, die oft als „theoriearme“ Methode der Brückenhypothesenkonstruktion bezeichnet wird, besteht in der empirischen Erhebung von Zielen oder Präferenzen, sei es durch Umfragen, Interviews oder explorative Studien (Kelle 1994, Opp/Friedrichs 1996). In der Tat muss jede Untersuchung, die über eine Restriktionsanalyse hinaus- geht, die Nutzenargumente irgendwie empirisch erheben. Doch klar ist auch, dass die theoriearme Methode keine Lösung des hier relevanten Problems darstellt. Siegwart Lindenberg hat überzeugend dargelegt, dass die Artikulation der Präferenzen keine Garantie für ihre kausale Relevanz verbürgt (Lindenberg 1996a: 130). Da jede Erhebung eine Mehrzahl von Präferenzen zutage fördert, stellt sich die Frage nach ihrer Bedeutsamkeit. Und diese Frage kann wiederum entweder ad-hoc oder theoretisch ${ }^{2}$ beantwortet werden (Lindenberg 1996a: 132).

Übrig bleibt damit nur die dritte, von Lindenberg selbst vorgeschlagene Strategie. In seinem „theoriereichen " Ansatz der Brückenhypothesenkonstruktion wird für ein strikt nomologisches Vorgehen plädiert, indem eine „Theorie der Präferenzen“ oder „Theorie der Ziele“ entwickelt wird. Doch Lindenbergs Vorschlag wurde scharf kritisiert (Kelle/Lüdemann 1995, Opp/Friedrichs 1996) und gilt seither als „umstritten“ (Greve 2006: 17).

Vor dem Hintergrund dieser unbefriedigenden Lage geht die vorliegende Untersuchung wie folgt vor: Zunächst wird Lindenbergs Modell erneut einem Test auf Konsistenz und Leistungsfähigkeit ausgesetzt, um mehr Klarheit über die Argumentationskraft der Kritik zu schaffen (2.1). Dabei wird gezeigt, dass Lindenberg eine spezifische „Theorie der Ziele“ bzw. eine „Theorie der Präferenzen“ entwickelt hat, die eine kreative Anwendung des Nachfragegesetzes auf die Relation zwischen Präferenzen und Metapräferenzen darstellt. In der ansatzimmanenten Kritik (2.2) wird allerdings gezeigt, dass die „Theorie der Ziele“ die Ad-hoc-Annahmen nicht eliminiert, sondern nur an eine andere (verdeckte) Einsatzstelle verschiebt. Ansatztranszendent (2.3) wird die Reduktion der Ziele menschlichen Handelns auf zwei „Grundbedürfnisse“ und die Annahme einer generellen Nutzenproduktionsfunktion kritisiert, die zur generellen Substitutionsannahme und infolgedessen zum Autonomieentzug für soziale „Handlungsräume“, „Sinnbereiche“ oder "Lebensordnungen" führt. Damit wird zugleich die schon von Parsons vertretene These bestätigt, dass ein ökonomischer Ansatz „strukturell“

${ }^{2}$ Ein Versuch, das Problem mit Hilfe statistischer Analyseverfahren zu lösen, würde auf einen Zirkel hinauslaufen: Der Sinn der Brückenannahmen besteht darin, sinnadäquate Hypothesen zu formulieren, die dann auf ihre empirische Adäquanz statistisch getestet werden sollen. Würde man bei der Konstruktion der Hypothesen auf statistische Verfahren vorgreifen, hätte man Sinnadäquanz durch empirische Adäquanz ersetzt. 
ungeeignet ist, eine gehaltvolle Theorie der Ziele zu entwickeln. Der institutionentheoretische Ansatz (3) schlägt einen anderen Weg ein. Darin wird die Autonomie der Lebensordnungen nicht als Hindernis, sondern als Chance für eine theoriegeleitete Konstruktion von Brückenhypothesen betrachtet. Gleichwohl wird auch die Einsicht in das Substitutionsverhalten nicht preisgegeben. Vielmehr wird die zentrale Aufgabe des Modells darin gesehen, gehaltvolle Aussagen darüber zu formulieren, wann ein Substitutionsverhalten, d.h. eine Anpassung des Handelns an die Restriktionen und Opportunitäten, und wann eine umgekehrte Handlungsanpassung an ein normatives Ideal einer Lebensordnung gegen variable Anreize, Opportunitäten und Restriktionen zu erwarten ist (3.1-3.3). Das Modell beruht also auf der Unterscheidung zwischen zwei Anpassungsrichtungen des Handelns (direction of fit). Auf der Grundlage dieser Unterscheidung wird ein konstellationsanalytisches Verfahren zur Gewinnung von Brückenhypothesen vorgeschlagen (3.4).

\section{Theoriereiche Konstruktion von Brückenhypothesen}

\subsection{Explikation des Modells}

Das Problem der Brückenhypothesen ergibt sich aus der Annahme der Vielfalt menschlicher Ziele, Zwecke oder Präferenzen. Wer davon ausgeht, dass nicht mit jeder Handlung sämtliche Ziele realisiert werden können, dem stellt sich die Aufgabe zu bestimmen, welches Ziel mit einer konkreten Handlung verfolgt wird. Im Rahmen einer subjektiven Werterwartungstheorie (SEU), die Siegwart Lindenberg vertritt, besteht die Aufgabe darin, „die entsprechenden Güter oder Ziele in die Nutzenfunktion einzugeben“ (Lindenberg 1996b: 129). Lindenbergs Strategie besteht in der Entwicklung eines theoretischen Modells zur Generierung von Brückenhypothesen, und zwar eines Modells, das den nomologischen Kern integrieren würde. Wie kann dies geleistet werden?

Lindenbergs Modell lässt sich auf folgende Überlegung zurückführen: ${ }^{3}$ Zwar kann eine Handlung A beliebige Ziele oder Präferenzen realisieren, die dem wissenschaftlichen Beobachter nicht bekannt sind, wenn es aber gelingen würde:

\footnotetext{
${ }^{3}$ Bei der nachfolgenden Darstellung handelt es sich nicht um eine Wiedergabe, sondern um eine Rekonstruktion des Ansatzes.
}

1) die Ziele oder Präferenzen sozialen Handelns dergestalt zu relationieren, dass sie nach einem (oder einigen) Kriterium(-en) miteinander vergleichbar wären (d.h. zu zeigen, dass sich hinter dem augenscheinlichen Chaos partikularer Präferenzen, Ziele oder Wünsche doch eine „Struktur" verbirgt),

2) die vorliegende Handlung A in Beziehung zu den relationierten (und daher vergleichbaren) Zielen zu setzen,

3) eine Selektionsregel anzugeben, nach welcher die relationierten, miteinander vergleichbaren Ziele selektiert werden,

dann könnte man aus einer vorliegenden Handlung, bei Kenntnis potenzieller Ziele und ibrer Relationen, aufgrund der Selektionsregel auf das Ziel von A schließen. Damit Lindenbergs Modell funktioniert, müssen die Bedingungen 1 bis 3 tatsächlich erfüllt werden.

ad 1. "Struktur" der Ziele - instrumentelle und universelle Präferenzen

Im neoklassischen Modell haben Präferenzen keine erkennbare „Struktur“, und zwar aus dem einfachen Grund, weil über Präferenzen grundsätzlich keine Aussagen getroffen werden. Anders gestaltet sich die Lage in dem von Stigler und Becker entwickelten Ansatz, der zwischen universellen und instrumentellen Präferenzen unterscheidet (Stigler/Becker 1977): „(...) assume general human goals and then look at all other goods as means for reaching these goals" (Lindenberg 1992: 132). Das hier wichtige Argument lautet, dass die konkreten Präferenzen in instrumentellen Beziehungen zu universellen Präferenzen stehen. Was muss man sich darunter genau vorstellen?

Lindenberg geht mit Stigler und Becker vom Menschenbild eines nutzenproduzierenden Akteurs aus (Lindenberg 1990b: 741). Dieser Akteur ist zwar auch ein Nutzenmaximierer, aber seine (für die Theorie der Ziele) wesentliche Eigenschaft ist die Nutzenproduktivität. Dabei geht es nicht um die Konsumption, also die augenblickliche Befriedigung von aktuellen Bedürfnissen, sondern um die Produktion von Zwischenprodukten, die als Mittel zur Produktion von universellen Präferenzen dienen. Die konkreten Präferenzen einer Handlung, z.B. der typischen familiären Einkaufstour am Samstagvormittag, sind nicht einfach aktuelle Begehrlichkeiten, Bedürfnisse oder Konsumptionswünsche, sondern Mittel zur Erreichung weiterer Zwischenziele, z.B. der Veranstaltung einer Geburtstagsparty, zu der zahlreiche Verwandte eingeladen werden, der Aufrechterhaltung der psy- 
chischen und physischen Kondition der Familienangehörigen, die wiederum für das berufliche Vorwärtskommen oder für den Abschluss der Ausbildung als notwendig erachtet werden etc. Mit dieser Überlegung lässt sich die zunächst unüberschaubare Vielfalt von Präferenzen, Zielen und Nutzenvorstellungen in eine „Ordnung“ bringen, die aus instrumentellen Produktionsketten besteht. Doch damit ist die Konstruktion noch nicht perfekt. Es fehlt die Annahme, dass die instrumentellen Produktionsketten in einem Punkt zusammenlaufen, dass alle Zwischenziele also selbst nur Mittel sind zur Produktion des abstakten Endproduktes: des Nutzens.

Man kann sich diese „Struktur“ als eine Pyramide vorstellen, deren Spitze der abstrakte Nutzen bildet, deren zweithöchste Ebene aus universellen Präferenzen besteht, die sich in instrumentellen Ketten weiter nach unten verzweigen (Kelle/Lüdemann 1995: 255ff.). Lindenberg möchte den bei Stigler und Becker substanzlosen Begriff der universellen Präferenzen mit konkreten Inhalten füllen. In Anlehnung an Adam Smith schlägt er vor, von zwei universellen „Oberzielen“ menschlichen Handelns auszugehen: vom physischen Wohlbefinden (PW) und von sozialer Anerkennung oder Wertschätzung (SW; Lindenberg 1990b: 741f., 1992: 132). Die beiden Oberziele zerfallen in sechs instrumentelle Hauptziele: „Das Kernstück der Hierarchie sozialer Produktionsfunktionen besteht aus den drei instrumentellen Hauptzielen für physisches Wohlbefinden (interner Komfort, externer Komfort und Aktivation) und den drei instrumentellen Hauptzielen für soziale Wertschätzung (Status, Verhaltensbestätigung und positiver Affekt) “ (Lindenberg 1996b: 135; vgl. Lindenberg 1992: 139f.). Die Ketten der instrumentellen Relationen sind also mindestens dreiteilig gegliedert und in ihrer konkreten empirischen Gestalt deduktiv nicht bestimmbar. Lindenberg selbst hat ein verbreitetes Missverständnis seines Ansatzes, man „könne Präferenzen aus der Theorie ableiten ", energisch korrigiert (Lindenberg 1996a: 563). ${ }^{4}$ Die instrumentellen Ketten von Zielen und Mitteln können „nach unten“ empirisch beliebig lang sein: „Je niedriger man in der Hierarchie ist, desto vielfältiger sind die Wege zu den Hauptzielen der Nutzenfunktion, also desto idiosynkratischer sind die Produktionsmittel " (Lindenberg 1996b: 135; vgl. Schmid 2004: 159).

\footnotetext{
${ }^{4}$ Die konkreten empirischen Produktionsfunktionen werden nicht abgeleitet, sondern durchaus empirisch erforscht. Aber nicht alle sozialen Institutionen und Produktionsfunktionen werden dabei vom Forscher rekonstruiert, sondern nur diejenigen, welche für die Realisierung theoretisch bestimmter Präferenzen von Bedeutung sind.
}

Die zentrale Bedeutung des Pyramidenmodells liegt darin, dass die konkreten Präferenzen nun nicht nur „strukturiert“, sondern auch hinsichtlich der beiden Oberziele relationiert sind. Auf diese Weise lässt sich jede konkrete empirische Präferenz mit jeder beliebigen anderen Präferenz vergleichen in Bezug auf die Frage, was sie zur Produktion der obersten Ziele beiträgt. Man kann sich Präferenzen/ Mittel vorstellen, die wenig zur Produktion von z.B. sozialer Anerkennung beitragen oder deren Beitrag weniger stabil und vorhersehbar ausfällt, und Präferenzen/Mittel, die besonders effizient und stabil sind. Damit kann die erste Vorbedingung für das Modell als erfüllt gelten. Bevor genauer auf die Frage der Präferenzenselektion eingegangen wird, muss die zweite Vorbedingung des Modells untersucht werden.

\section{ad 2. Die Beziehung von Handlung und Zielstruktur}

Die zweite Vorbedingung besteht darin, die vorliegende Handlung A in Beziehung zu den relationierten (und daher vergleichbaren) Zielen oder Motiven zu setzen. Denn aus dem Vorliegen selbst einer detaillierten und universell geltenden Präferenzenhierarchie folgt keineswegs, welche Präferenz A zugrunde gelegt wurde, und dies aus zwei Gründen: Erstens, weil immer noch gilt, dass jede Handlung multifunktional sein kann, d.h. sie kann an unterschiedlichen Stellen an die Präferenzenhierarchie „anschließen“. Der zweite Grund ist logischer Natur. Ziele oder Präferenzen sind keine Handlungsanweisungen. Selbst wenn wir wissen, dass X eine Universitätskarriere anstrebt, können wir nicht sagen, was er konkret dafür zu tun beabsichtigt. Das Modell der Handlungserklärung muss also die Zieloder Präferenzenhierarchie logisch mit den Handlungsweisen verknüpfen.

Diese Aufgabe ist allerdings vor dem Hintergrund des Begriffs der instrumentellen Präferenz nicht allzu schwer zu lösen. Denn der Begriff hat bei Stigler/Becker, ebenso wie bei Lindenberg, ein Doppelgesicht: Er bezeichnet sowohl Ziele als auch Handlungsmittel. Damit wird deutlich, was Lindenberg mit der Aussage meint, dass die instrumentellen Präferenzen im technischen Sinne als Restriktionen des Handelns erscheinen (Lindenberg 1990b: 741, 1996b: 135, Schmid 2004: 159). Der Vollzug von A ist für X sowohl Ziel als auch Mittel zur Erreichung weiterer Ziele.

Was aber verbirgt sich konkret hinter dem Begriff „Mittel“ oder „Produktionsmittel“? Nach Lindenberg sind es soziale Institutionen und/oder Organisationen. Die Realisierung der Präferenz für die 
Wissenschaftskarriere erfordert z.B. die Institutionen der Universität und der wissenschaftlichen Öffentlichkeit. Die Realisierung der Präferenz für soziale Anerkennung kann vermittels der Institution der Familie, der Freundschaftsbeziehung oder im Rahmen einer zivilgesellschaftlichen Organisation erfolgen. „So bringt soziale Wertschätzung Nutzen, ist Status ein Mittel zur Produktion sozialer Wertschätzung, ist ein Lehrstuhl ein Mittel, um Status $\mathrm{zu}$ erreichen, sind die Promotion und zahlreiche wissenschaftlichen Publikationen Mittel, einen Lehrstuhl zu erhalten usw" (Lindenberg 1996b: 135). Soziale Institutionen und Beziehungen haben für die Akteure die Funktion, einen bestimmten Nutzen zu produzieren. Hinter Lindenbergs zentralem Begriff der sozialen Produktionsfunktion (SPF) verbergen sich also soziale Beziehungen und Institutionen unter dem Aspekt ihrer Beiträge zur Herstellung von universellen Gütern (Esser 2000a: 91ff.). Wenn Menschen Institutionen oder soziale Beziehungen in Anspruch nehmen, herstellen oder in sie investieren, dann tun sie es, um bestimmte Präferenzen zu verwirklichen. Der Begriff der Produktionsfunktion verknüpft also Handlungen und Präferenzen. Erst vor dem Hintergrund der in einer Gesellschaft verfügbaren Produktionsfunktionen verstehen wir, warum ein Anwärter auf eine Universitätskarriere seine Zeit z.B. nicht damit verbringt, Freunde in hochschulpolitischen Ämtern zu gewinnen, sondern wissenschaftliche Publikationen zu schreiben. „Von einer Kultur kann man sagen, daß sie charakteristische soziale Produktionsfunktionen für verschiedene soziale Positionen in verschiedenen sozialen Situationen hat" (Lindenberg 1990a: 272).

Der Vorteil dieser Konzeptualisierung gegenüber dem theoriearmen Ansatz lässt sich auf zwei Punkte zurückführen: Erstens werden die Präferenzen nicht als sich dem sozialwissenschaftlichen Zugriff entziehende subjektive Dispositionen oder Wünsche aufgefasst, sondern als intersubjektive, institutionalisierte „Produktionsmittel“, die der sozialwissenschaftlichen Analyse zugänglich sind (Lindenberg 1996a: 562). Lehrstühle, politische Ämter, Freundschaftsbeziehungen, Bildungspatente oder Berufe bestehen aus intersubjektiv anerkannten Handlungsregeln, über welche die Befragten unabhängig von ihren momentanen subjektiven Befindlichkeiten Auskunft geben können. Zweitens wird es deutlich, dass die empirische Erhebung der Präferenzen, von der die Anhänger des theoriearmen Ansatzes reden, nur vor dem Hintergrund institutionalisierter Regeln Erfolg haben kann. Denn nur diese verbürgen, dass nicht beliebige Präferenzen, sondern nur Präferenzen, die in institutionalisierten Sinnzusammenhängen mit den zu erklärenden Handlungen stehen, abgefragt werden. Ansonsten könnte man Abläufe von Universitätskarrieren auch mit religiösen oder kulinarischen Präferenzen erklären wollen. Lindenberg wendet sich also nicht generell gegen die empirische Erhebung der Präferenzen, sondern nur gegen die Illusion, die „Messung“ könne die Erklärung ersetzen.

Damit wird die zweite Vorbedingung für die Aufstellung der Theorie der Ziele erfüllt. Lindenberg hat nicht nur eine universelle Struktur der Präferenzen entwickelt, sondern diese durch den Begriff der Produktionsfunktion auch in Beziehung zum Handeln gesetzt.

\section{ad 3. Die Selektionsregel}

Allerdings ist damit das Erklärungsmodell immer noch nicht perfekt. Man kann sich davon überzeugen, wenn man überlegt, wie weit ein Beobachter, ausgerüstet allein mit den analytischen Instrumenten der Zielstruktur und den Produktionsfunktionen, bei der Erklärung von A kommen würde. Er würde, erstens, annehmen, dass A letztlich der Erreichung eines der beiden (oder beider) Oberziele dient. Er würde, zweitens, überlegen, zu welchen Institutionen A überhaupt potenziell passt. Dazu wird er auf die kulturellen (Frames) und institutionellen Aspekte der SPFs eingehen. Er wird z.B. feststellen, dass das Verfassen einer wissenschaftlichen Publikation im institutionellen Kontext des Wissenschaftsbetriebs steht, und daraus schließen, dass der Verfasser eine Universitätskarriere anstrebt, die aber letztlich dem Oberziel der sozialen Anerkennung dient. Ein sozialwissenschaftlich geschulter Beobachter wird sich aber mit dieser Hypothese nicht zufriedengeben. Denn die Publikation in einer Fachzeitschrift steht prima facie im institutionellen Kontext des Wissenschaftsbetriebs, aber dieser bleibt mit anderen institutionellen Feldern verbunden. Man kann mit wissenschaftlichen Publikationen auch politische oder wirtschaftliche Ziele verfolgen. Handelt es sich bei dem Verfasser um den amtierenden Außenminister oder um einen in der Forschungsabteilung einer Pharmafirma angestellten Wissenschaftler, dann gewinnen derartige alternative Hypothesen an Plausibilität. Denkbar ist also, dass die Publikation nicht durch die Erfordernisse einer wissenschaftlichen Karriere, sondern politisch, wirtschaftlich oder "intrinsisch “ motiviert ist. Der Beobachter ist in der Lage, A in vielen potenziellen institutionellen Kontexten zu verorten, diese den Produktionsfunktionen zuzuordnen; er ist jedoch nicht in der Lage vorauszusagen, welche der 
vielen potenziell möglichen Produktionsziele A tatsächlich verursacht hat.

Um dies zu bestimmen, benötigt der Beobachter eine Selektionsregel. Die Neoklassik verfügt mit dem Nachfragegesetz über eine solche Selektionsregel. Sie erklärt die Variation des Handelns aus der Veränderung relativer Preise. Doch die Preise beziehen sich im neoklassischen Forschungsprogramm auf die Objekte des Handelns und nicht auf die Präferenzen, die das Handeln steuern.

Es ist ein kühner Vorschlag von Lindenberg, das neoklassische Nachfragegesetz auf die Präferenzen oder Ziele sozialen Handelns zu beziehen, also gleichsam nach dem „Preis“ der Präferenzen zu fragen. Das Terrain für dieses Manöver ist allerdings mit den Annahmen der hierarchischen und instrumentellen Präferenzen bereits vorbereitet (Stigler/ Becker 1977). Die „Pyramidenstruktur“ der Ziele macht es möglich, dass ein Zwischengut auf unterschiedliche Weise produziert wird. ${ }^{5}$ Wenn ein und dasselbe Produkt in unterschiedlichen Produktionsfunktionen hergestellt werden kann, dann sind die (Zwischen-) Produktionsfunktionen substituierbar. Ist das der Fall, lassen sie sich auch nach ihren relativen Preisen auswählen. „Wenn beispielsweise Status im Moment nicht viel zur sozialen Wertschätzung einer Person beiträgt (etwa weil sie dem Berufsleben durch langfristige Arbeitslosigkeit oder Pensionierung enthoben ist), dann scheint es so, als nähme im Wertgefüge dieser Person Status einen sehr niedrigen Platz ein. Tatsächlich aber ist es für diese Person sehr ineffizient, soziale Wertschätzung durch Suche nach Status zu gewinnen “ (Lindenberg 1996a: 562). Die marginalen Kosten der Inanspruchnahme der Produktionsfunktion „Beruf“ sind für einen Langzeitarbeitslosen so hoch, dass er das Oberziel der sozialen Anerkennung lieber über günstigere Produktionsfunktionen, etwa über eine Verhaltensbestätigung oder einen positiven Affekt, zu erreichen versucht. ${ }^{6}$ Im Kern geht es bei Linden-

\footnotetext{
${ }^{5}$ Lindenberg modelliert die Selektion folgerichtig im Rahmen einer Cobb-Douglas-Funktion, welche die Produktivität vom Einsatz mehrerer Produktionsfaktoren abhängig macht. „Sie lautet für den Fall der Produktion eines Gutes $\mathrm{X}$ durch zwei Faktoren $\mathrm{V}_{1}$ und $\mathrm{V}_{2}$ allgemein $\mathrm{x}=\mathrm{Av}_{1}{ }^{\alpha_{\mathrm{V}}} \mathrm{v}_{2}$ b، (Esser 2000b: 49).

${ }^{6}$ Als weiteres Beispiel für die Substitution des Status und der Verhaltensbestätigung durch Affekt wird von Lindenberg die Entscheidung über das Übersiedeln in ein Altersheim angeführt. „Im Älterwerden fallen bei alten Leuten eher mehr die Alternativen Status und Verhaltensbestätigung weg, so daß die Produktion physischen Wohlbefindens eher mehr auf Komfort und die Produktion sozialer Wertschätzung eher mehr auf Affekt ausgerichtet ist. So-
}

bergs theoriegesteuerter Konstruktion von Brückenhypothesen um eine Restriktions- und Opportunitätsanalyse: „Ganz allgemein suchen wir bei jedem Individuum nach den Umständen, unter denen es mehr oder weniger regelmäßig soziale Wertschätzung und physisches Wohlbefinden produziert" (Lindenberg 1996a: 562; Hervorhebung M.S.). „Je größer die Effizienz eines Produktionsfaktors für die Befriedigung der Grundbedürfnisse innerhalb des jeweiligen Kontextes, desto eher wird dieser Produktionsfaktor gewählt" (Nauck 2001: 413). Lindenberg „kopiert“ gewissermaßen den neoklassischen Ansatz, der ursprünglich auf der Ebene der Handlungsobjekte angesiedelt war, auf die Ebene der Präferenzen, um des Problems der selektiven „Multifunktionalität“ des Handelns Herr zu werden.

Mit der Einführung der Selektionsregel ist das Modell der theoriegesteuerten Konstruktion von Brückenhypothesen komplett. Um herauszufinden, welches Ziel A bestimmt, muss man nach den relativen Preisen und Effektivitäten der relevanten Produktionsfunktionen zur Herstellung von Ober- und Hauptzielen für den Akteur fragen. Ein amtierender Außenminister hat es nicht nötig, auf wissenschaftliche Institutionen zurückzugreifen, um sozialen Status zu erlangen. Ihm stehen ganz andere, viel preiswertere und effektivere Produktionsfunktionen zur Verfügung. Seine Präferenzen sind also im politischen Bereich zu suchen. Anders sieht es bei einem „hauptberuflichen“ wissenschaftlichen Mitarbeiter aus, der bei der Produktion von Status auf eine Universitätskarriere angewiesen ist, und noch anders bei einem Privatgelehrten, der zwar vermutlich ebenfalls bestrebt ist, sozialen Status zu erlangen, aber nicht qua Wissenschaftsbetrieb. Das Modell erlaubt es also, aus den Zugangsmöglichkeiten des Akteurs zu unterschiedlichen Produktionsfunktionen Rückschlüsse auf die Präferenzen seiner beobachtbaren Handlungen zu ziehen. Der Sinn der Lindenbergschen Konstruktion besteht darin, eine Alternative zur bloßen Restriktionsanalyse zu entwickeln, die in ihrer selbst gewählten Abstinenz darauf verzichtet (und es nicht für nötig hält), die Vielfalt der Ziele eines Handelns aufzudecken. Gegen diese immer nur partielle, an die relativen Schwankungen der Preise angelehnte Erklärung des Handelns richtet sich der Ansatz der theoriereichen Brückenannahmen. Es geht dabei nicht darum, den Einfluss variierender Preise einer mehr oder weni-

lange Verluste in den Produktionsmitteln dieser beiden Güter kompensiert werden können, ziehen Menschen das selbstständige Wohnen vor“ (Lindenberg 1996b: 138). 
ger beliebig gewählten Produktionsfunktion auf das Handeln zu bestimmen, sondern darum zu sagen, welche Produktionsfunktionen das Handeln bestimmen.

\subsection{Ansatzimmanente Kritik}

Lindenbergs Ansatz wurde bereits ausgiebig kritisch diskutiert. ${ }^{7}$ Die Diskussion vermittelt ein zwiespältiges Bild. Auf der einen Seite konnten die Kritiker den Ansatz vor allem in seinen forschungspraktischen Aspekten weitgehend entzaubern. Auf der anderen Seite hat man nicht den Eindruck, dass sein theoretischer Kern getroffen wurde. ${ }^{8}$ An dieser Stellte soll eine entsprechende ansatzimmanente Kritik entwickelt werden.

Um das Ziel einer Handlung zu erklären, benötigt das Modell Informationen über die vom Akteur gewählte Güterkombination, über die Restriktionen (Preise und Einkommen) und die Effizienzen der Produktionsfunktionen. Die These der vorliegenden Abhandlung lautet nun: Der Versuch, die Effizienz der sozialen Produktionsfunktionen zu bestimmen, führt in die eingangs geschilderte, problematische Ausgangsposition zurück, die in der Alternative von Ad-hoc-Annahmen und empirischer Ermittlung von Präferenzen besteht. Worin liegt hier die Schwierigkeit?

\footnotetext{
${ }^{7}$ Vgl. die Auseinandersetzung zwischen Siegwart Lindenberg auf der einen und Udo Kelle, Christian Lüdemann, Karl-Dieter Opp sowie Jürgen Friedrichs auf der anderen Seite, die im Forum der Kölner Zeitschrift für Soziologie und Sozialpsychologie ausgetragen wurde (Kelle/Lüdemann 1995, Lindenberg 1996a, 1996b, Lüdemann/Rothgang 1996, Opp/Friedrichs 1996).

${ }^{8}$ Opp und Friedrichs haben Lindenberg darüber hinaus vorgeworfen, sein Ansatz könne keine „Ableitung“ von Brückenannahmen bewerkstelligen, und insofern unterscheide er sich in der praktischen Vorgehensweise kaum von theoriearmen Ansätzen (Opp/Friedrichs 1996: 551). Dieses Argument konnte Lindenberg jedoch mit guten Gegenargumenten zurückweisen: Das Modell bezweckt keine „Ableitung“ konkreter empirischer Motive oder Institutionen, sondern nur die Aufstellung von „Suchheuristiken “ für die Identifikation jener empirischen Motive, die mit den theoretisch bestimmten Brückenannahmen korrespondieren. Der theoretische Ansatz kann und will nicht sagen, wie Menschen konkret-empirisch z.B. „Status“ erlangen, sondern dem Forscher nur Hinweise geben, dass nach empirischen Motiven und Institutionen für die „Statusproduktion“ gesucht werden soll (Lindenberg 1996a: 562). Wir sind „schon einen Riesenschritt weiter“, wenn wir wissen, nach welchen instrumentellen Mitteln wir suchen (Lindenberg 1996a: 563).
}

Der Begriff der Effizienz stammt aus der Produktionstheorie, die „das Verhalten von Unternehmern, die unter Einsatz von Produktionsfaktoren bestimmte Güter erzeugen und dann auf einem Markt verkaufen“, erklärt (Esser 2000b: 59). Die Effizienz einer Produktionsfunktion bezeichnet das Verhältnis zwischen den Mengen eingesetzter Produktionsfaktoren und der Menge des erzeugten Gutes. Die Bestimmung der Effizienz einer Funktion ist nicht problematisch: Man muss lediglich wissen, was für die Produktion eingesetzt wurde und was dabei herauskam. Das Wissen über die Effektivität der Produktion ist darüber hinaus in instrumentellen Handlungsregeln gespeichert. Man kann sie also auch aus der Analyse der gesellschaftlich anerkannten, technischen Regeln gewinnen. Was in der Wirtschaft funktioniert, kann auch in anderen Bereichen sozialen Handelns funktionieren. So weiß z.B. ein Wissenschaftler (in grober Annäherung), wie effektiv der Einsatz von Arbeit, Wissen und Zeit für die Herstellung einer wissenschaftlichen Publikation ist. Auch die Effizienzen der Produktion weiterer indirekter Zwischengüter in der Kette: Publikationen - Promotion - Lehrstuhl lassen sich grundsätzlich bestimmen. ${ }^{9}$ So weit, so gut. Doch um die Effizienz einer SPF zu bestimmen, reicht es nicht aus, die „realen“ Produktionsschritte (Publikationen Promotion - Lehrstuhl) in Betracht zu ziehen. Dies sind lediglich indirekte und primäre Zwischengüter, die eingesetzt werden, um die Oberziele (PW, SW) zu erzeugen. Die Bestimmung der Effizienz einer SPF setzt darüber hinaus voraus, dass man auch die Effizienz der Produktion von Oberzielen und schließlich die Effizienz der Endnutzenfunktion selbst angeben kann. ${ }^{10}$

Doch bei der „Produktion“ von Oberzielen und der „Produktion“" von Nutzen handelt es sich nicht mehr um institutionell geregelte Vorgänge in der sozialen Wirklichkeit, sondern um Vorgänge „in den

\footnotetext{
${ }^{9}$ Das heißt, es gibt keine theoretischen Argumente gegen die Möglichkeit der Bestimmung der Effizienz des institutionellen Handelns. Aber: "Je verschachtelter Produktionsfunktionen sind, d.h. je eher instrumentelle ,Zwischenziele nötig sind, um die ,höchsten` Ziele zu realisieren (...), desto schwieriger dürfte es sein, die ,wahren' Produktionsfunktionen zu rekonstruieren." (Kelle/ Lüdemann 1995: 257)

${ }^{10}$ Und um unterschiedliche Produktionsfunktionen miteinander zu verknüpfen, muss man davon ausgehen, dass sie der Herstellung eines Produkts dienen. Ohne den Endproduktbezug laufen die Fäden der Produktionsketten nicht auf einen zentralen Punkt zu, sondern in alle Himmelsrichtungen auseinander; die Selektionsregel greift ins Leere.
} 
Köpfen“ der Akteure. Die Effizienz dieser Vorgänge lässt sich nicht durch die Analyse institutioneller Handlungsregeln bestimmen. Eine Institution verlangt ein bestimmtes Verhalten in einer bestimmten Situation („Verträge sind einzuhalten“) oder legt bestimmte positive oder negative Sanktionen für ein situativ gefordertes Verhalten fest („ein unparteiischer Richter genießt hohes Ansehen“). Diese Sanktionen können für die betroffene Person auch nützlich sein. Doch welchen Anteil diese Belohnung an der Nutzenproduktion hat, in welcher Relation diese Belohnung zu anderen nützlichen Handlungen oder Belohnungen steht, darüber sagen Institutionen nichts aus. Inwieweit ein Richter- oder Priesteramt, ein Lehrstuhl, ein Management- oder Gewerkschaftsposten zum physischen oder psychischen Wohlbefinden der betroffenen Person beiträgt, steht in keiner institutionellen Regelung.

Wie wird mit diesem Problem im Rahmen des Modells der SPF umgegangen? Schaut man sich die Anwendungsbeispiele bei Siegwart Lindenberg und Hartmut Esser an, so kommt man rasch zu dem Befund, dass es sich dabei allesamt um Annahmen handelt. Im Lehrbuchbeispiel von Esser werden die Koeffizienten zweier Produktionsfaktoren einfach im Verhältnis 50:50 (Esser 2000b: 76) oder, zur Veranschaulichung unterschiedlicher Präferenzen der Akteure, im Verhältnis 80:20 und 20:80 angenommen (Esser 2000a: 100, 2000b: 86). Was für pädagogische Zwecke höchst sinnvoll sein kann, das reicht für Forschungszwecke kaum aus. Soll man annehmen, dass die wichtigsten SPFs, etwa Familie, Beruf und Freizeit, mit je $33 \%$ an der Erzeugung von Oberzielen und diese wiederum mit $50 \%$ an der Erzeugung des Endnutzens beteiligt sind? Kommen weitere SPFs hinzu, etwa Religion, Sport, Freudenkreis, Kunst oder Politik, sollen dann die Effizienzen von Beruf und Familie auf je $20 \%$ reduziert werden? Es ist allzu offensichtlich, dass generelle Annahmen über die Effizienzen unterschiedlicher SPFs willkürlich ausfallen müssen. Als Alternative bleiben lediglich fragestellungsspezifische, d.h. letztlich Ad-hoc-Annahmen übrig.

Damit zeigt sich, dass Lindenbergs Modell der theoriereichen Konstruktion von Brückenhypothesen die Ad-hoc-Annahmen nicht eliminiert, sondern nur an eine andere, weniger offensichtliche Einsatzstelle verschoben hat. Statt unmittelbarer Präferenzen einer Handlung werden jetzt Effizienzen der Erzeugung von Endnutzen durch den Einsatz von unterschiedlichen institutionalisierten Produktionsmitteln unterstellt. Es ist also das letzte (und vorletzte) Glied in der Produktionskette, das sich beim näheren Betrachten als Versteck von „adhocness“ entpuppt. Lindenbergs Ansatz wurde häufig wegen der Reduktion der menschlichen Ziele oder Bedürfnisse auf PW und SW kritisiert. ${ }^{11}$ Diese ansatztranszendente Kritik ist, wie im Weiteren ausgeführt wird, berechtigt. An dieser Stelle konnte aber gezeigt werden, dass die Konstruktion auch ansatzimmanent kritisiert werden muss: Selbst wenn man die Lindenbergsche Reduktion der Bedürfnisse akzeptiert, ist sie aufgrund der willkürlichen Verknüpfung von Oberzielen mit den realen Produktionsketten angreifbar.

Man könnte nun des Weiteren fragen, ob sich die Effizienzen der Endnutzenfunktion vielleicht empirisch bestimmen lassen. Aber auch dieser Weg scheint nicht gangbar zu sein. Zwar ist Lindenberg nicht von dem Problem betroffen, mit dem der theoriearme Ansatz zu kämpfen hat: dem fehlenden Regelbezug der abgefragten Präferenzen/Effizienzen, der zu einem Erklärungsdefizit des Modells führt. Doch der bei Lindenberg durchaus vorhandene Regelbezug ist mit einem komplementären Fehler behaftet: Da seine Theoriekonstrukte in der Lebenswelt der Akteure nicht vorkommen, sind valide Antworten auf Fragen nach der Effizienz der Produktionsketten kaum zu erwarten. Lindenbergs Ansatz basiert auf Regeln ohne einen sozialen Sinnbezug. Der theoriearme Ansatz erfasst den sozialen Sinnbezug ohne einen Regelbezug. Der fehlende Regelbezug führt zu einem Erklärungsdefizit, der fehlende soziale Sinnbezug zu einem Verstehensdefizit.

\subsection{Ansatztranszendente Kritik}

Ein ansatzimmanentes Problem besteht bei Lindenberg in der Art und Weise, wie die Effizienzen der „letzten“ Nutzenfunktion bestimmt werden. Ansatztranszendent lässt sich kritisieren, dass das soziale Handeln im Rahmen einer Nutzenfunktion modelliert wird. Lindenberg bildet die Nutzenproduktion mit Hilfe der Cobb-Douglas-Funktion ab, die impliziert, dass der Nutzen unter dem Einsatz von mehreren Faktoren produziert wird. ${ }^{12}$ Der Endnutzen ist also immer das Produkt einer $\mathrm{Mi}$ -

\footnotetext{
${ }^{11}$ Die inhaltliche Festlegung sei „nicht theoretisch begründet (...), sondern beruht auf einer Setzung “ (Kelle/Lüdemann 1995: 256, Opp/Friedrichs 1996: 551). Würde man nicht von utilitaristischen Hintergrundannahmen ausgehen, könnte man mit genauso gutem Recht im psychischen Wohlbefinden und Machtstreben oder im Eros und Thanatos die Grundbezüge menschlicher Existenz sehen.

${ }^{12}$ Vgl. Fn. 6.
} 
schung von unterschiedlichen Gütern und Produktionsvorgängen, z.B. des Familienhandelns, der Berufskarriere und der sportlichen Aktivität, die in unterschiedlichen Effizienzgraden zur Herstellung von Endnutzen beitragen. Zwar sind die unterschiedlichen SPFs in dem Sinne nicht aufeinander reduzierbar - man kann nicht die eine Kette durch eine andere ersetzen -, aber sie sind alle miteinander verbunden. Ändert sich der Preis für einen bestimmen Produktionsfaktor (z.B. Beruf), dann hat das Auswirkungen auf alle Produktionsketten. Verteuert sich der Preis für die Produktion von Berufskarrieren, dann ändert sich das gesamte Budget des Akteurs und seine gesamte Investitionsstrategie, d.h. er wird nicht nur weniger in die Karriere investieren, sondern (ceteris paribus) auch mehr in Familie und Sport. ${ }^{13}$

Lindenberg vertritt also die Thesen einer generellen Substitutionsfähigkeit der Faktoren und einer generellen Verbundenheit aller Produktionsketten. Beide Thesen sind aber problematisch. Zweifellos gibt es Faktoren, die bei jeder Tätigkeit eingesetzt werden. Der wichtigste davon ist die Zeit. Aber nicht alle Faktoren sind substituierbar, und daher lassen sie sich nicht als Bestimmungsgrößen eines Budgets, eines Handlungsraums begreifen. Darum ist eine generelle Substitutionsunterstellung falsch: Die wirtschaftliche Entwicklung führt nicht in jeder Gesellschaft und nicht in jedem Kulturkreis zur Marginalisierung der Religion (Riesebrodt 2000); demokratisches Engagement der Bürger wird durch die Wohlstandssteigerung nicht gehemmt, sondern gefördert (Putnam et al. 1994); höhere Bildung muss nicht zwingend zur Instabilität der Partnerschaftsbeziehungen führen (Eckhard 2008); nicht jede Erweiterung des Handlungsraums hat Einfluss auf alle Produktionsfunktionen. Die Zwischengüter sind raumspezifisch einsetzbar, und die Konvertibilität der Kapitalsorten ist nur begrenzt und, wenn überhaupt, nur unter hohem Aufwand möglich (Bourdieu 2006: 194ff.). Ehe man also eine bestimmte Erweiterung oder Verengung des Handlungsraums als Ursache eines vermeintlichen Substitutionsverhaltens auffasst, muss man fragen, ob die gegebenen Zwischengüter überhaupt relevant für die fraglichen Produktionsvorgänge sind. Nicht alles, was in den

13 Ein anderer Kritikpunkt könnte lauten: Das Nachfragegesetz erlaubte bekanntermaßen, nur Restriktionseffekte, aber keine Opportunitätseffekte zu erfassen. Das heißt, man kann davon ausgehen, dass die Verteuerung einer Produktionsfunktion zur Folge haben wird, dass die Funktion weniger nachgefragt wird. Aber man kann nicht voraussagen, welche Effekte die Opportunitätserweiterung zeitigen wird.
Augen eines Substitutionstheoretikers als eine Opportunität erscheint, stellt für die Akteure in der sozialen Wirklichkeit tatsächlich eine Gelegenheit des Handelns dar. Damit soll nicht behauptet werden, dass so etwas wie Substitutionsverhalten nicht existiert. Vielmehr gibt es sowohl Anpassungen an neue Handlungsräume als auch Resistenzen der Produktionsfunktionen gegen Veränderungen der Restriktionen des Handelns. Die Aufgabe besteht darin, beides zu erfassen und zu erklären, indem man theoretisch Bedingungen für das Auftreten der einen oder der anderen Anpassungsrichtung bestimmt.

Die Kritik an der Annahme der generellen Verbundenheit aller SPFs über eine übergeordnete Nutzenproduktionsfunktion lautet: Diese Annahme konfligiert mit dem Mainstream soziologischer Differenzierungstheorien, die von autonomen „Systemen“, „Sinnprovinzen“ oder „Wertsphären“ ausgehen. Zwar wird in der Theorie der SPF von "Codes“ und „Frames“" gesprochen, welche die einzelnen SPFs zu kulturell autonomen „Sinnbereichen “ oder „Wertsphären“ aufrüsten sollen. Doch die Inkonsistenz dieser Redeweise zeigt sich bei der Frage, aus welchen Motiven die SPFs aufrechterhalten werden. „Da man soziale Wertschätzung nur über die Erfüllung der in den jeweiligen institutionalisierten Sphären geltenden Erfolgsbedingungen erlangen kann, hält man sich daran" (Schwinn 2006: 47). Doch genau dies ist äußerst problematisch. Die Orientierung an den sphärenspezifischen Frames und Codes kann nämlich in Konflikt mit dem Interesse an den Oberzielen geraten. Es ist keineswegs theoretisch begründbar, weshalb das Ausmaß der sphärenspezifischen Anforderungen genau der optimalen individuellen Strategie des Akteurs entsprechen soll. Aus der rationalen Entscheidung eines Ehemanns, aufgrund seiner Budgetlage und nach Abschätzung der Effizienz der SPFs etwa $20 \%$ seiner Ressourcen in das Familienleben zu investieren, folgt keineswegs, dass die Ehefrau auch genau diese $20 \%$ für sich beanspruchen muss. Es kann gut sein (und der Gesellschaftsroman des 19. Jahrhunderts, von Fontane bis Flaubert, liefert hierfür anschauliche Beispiele), dass sie aufgrund ihrer Budgetlage $40 \%$ von ihm verlangen wird. Der Ehemann wird also mit einer verstärkten Präsentation des Ehe-Frames konfrontiert, ohne ein Nutzeninteresse an der Anerkennung der darin enthaltenen Geltungsansprüche zu haben.

Damit wird deutlich, dass die Einhaltung der sphärenspezifischen Produktionsanforderungen, anders als im Modell der SPF angenommen, nicht notwendigerweise vom Eigeninteresse der Akteure getragen wird. Vice versa ist die Abweichung von den 
Codes und Frames nicht notwendig selbstschädigend. Sobald das Nutzeninteresse und die sphärenspezifischen Anforderungen auseinanderfallen, entsteht für den Akteur ein Anreiz, nicht den Codes und den Frames, sondern seiner subjektiv nutzenmaximierenden Strategie zu folgen. Dies kann die Form eines offenen Konfliktes oder einer Täuschung annehmen, was wiederum entweder zum Zerfall der Institutionen und zum Zusammenbruch der Ordnung oder zu einer ökonomisch motivierten Vertragsschließung führen muss. Beides ist gleichbedeutend mit der Aufhebung der genuinen Sinnautonomie der sphärenspezifischen Frames. Dieser Umstand wurde auch zum Anlass einer anhaltenden Kritik an der älteren Version der Framing-Theorie von Esser, die stärker ökonomisch fundiert war. ${ }^{14}$ Der springende Punkt dabei ist, dass im Konflikt zwischen dem subjektiven Nutzeninteresse und den Anforderungen sphärenspezifischer Frames Letztere immer den Kürzeren ziehen werden. Der Grund dafür liegt darin, dass sie selbst keine motivationale Kraft haben, dass sie den Akteur weder normativ binden noch beeinflussen können. Für den ökonomischen Ansatz sind „die Wertsphären und die entsprechenden Institutionen nur Zwischengüter, der eigentliche Antrieb und die Motivation kommt aus den beiden basalen Bedürfnissen des psychischen Wohlbefindens und der sozialen Wertschätzung. Geld, Liebe, Macht etc. sind nur Mittel zum Zweck, um den eigentlich interessierenden primären Nutzen zu erzeugen" (Schwinn 2006: 47). Die übergeordnete Nutzenproduktionsfunktion entzieht den einzelnen Bereichen normative und motivationale Eigenkraft. Frames sind in dieser Perspektive lediglich Such- und Informationsbearbeitungskosten reduzierende „Sparmaßnahmen“ (North 1988: 50). Es ist also ein normatives Geltungsdefizit, welches das Modell der SPF und die ältere Version der Theorie der Frame-Selektion von Hartmut Esser kennzeichnet (Stachura 2006).

Der Beitrag der SPF zur Generierung von Brückenhypothesen liegt vor allem in der Einsicht, dass Institutionen Geltungskontexte haben, durch die sie für die Handlungserklärung identifiziert werden können. Wertvoll ist auch die Einsicht in das Substitutionsverhalten der Akteure; möchte man jedoch

14 Ein Frame, der einen hohen Nutzen stiftet, wird demnach auch dann gewählt, wenn „die Wahrscheinlichkeit dafür, daß dies der ,richtige' Frame ist, relativ gering ist“ (Lüdemann/Rothgang 1996: 284, Rohwer 2003: 343, Srubar 1992: 163, Stachura 2006: 448). Eine Zusammenfassung der kritischen Einwände bietet Kroneberg (2005). Die neuere Version der Theorie der Frame-Selektion (Kroneberg 2007) behebt teilweise das Problem. der Autonomie der Wertsphären theoretisch Rechnung tragen, kann die These der generellen Substitution nicht aufrechterhalten werden. Vielmehr muss diese zur These der bedingten Substitution abgeschwächt werden, wie dies im Weiteren im Rahmen eines an Weber orientierten Ansatzes gezeigt werden soll.

\section{Institutionentheoretische Alternative}

In der kritischen Auseinandersetzung mit dem Modell der SPF sind die Differenzen zu der hier entwickelten Modellierung stichwortartig schon angeklungen: Vielfalt der Wertsphären statt Einheit der Dachnutzenfunktion, Autonomie der Sphären statt Heteronomie der Produktionsfunktionen, Wertverwirklichung statt Nutzenproduktion, bedingte Substitution statt genereller Substitutionsannahme, konstellationsanalytische statt nomologische Vorgehensweise bei der Konstruktion von Brückenhypothesen. Diese Stichpunkte sollen im Weiteren ausgeführt werden.

In der weberianischen Theorietradition wird das Handeln nicht als Nutzenproduktion, sondern als Verwirklichung von Werten aufgefasst. Ein wichtiger Unterschied besteht hier darin, dass Werte um ihrer selbst willen realisiert werden, während der Nutzen einer Handlung immer einen weiteren Zweck voraussetzt, um dessentwillen die Handlung vollzogen wird. Während Lindenberg alle Produktionsketten lediglich als Mittel begreift, sind bei Max Weber „dagegen die sphärenspezifischen Werte nicht Mittel zum Zweck, sondern Selbstzweck, ein Wert an sich, um dessen willen man handelt. Diese Endzwecke dienen ihrerseits nicht nochmals als Zwischenglieder einem weiteren Ziel oder Zweck “ (Schwinn 2006: 47). Da Menschen fähig sind, verschiedene Werte gleichzeitig als letztgültige anzuerkennen, gibt es in der sozialen Wirklichkeit mehrere irreduzierbare Wertsphären und Ordnungen (Schwinn 2001: 187, 191). Die Anzahl dieser Werte ist nicht a priori festlegbar: „Unsere Annahmen darüber, welche Handlungsgründe, Ziele und Werte als letzte anzuerkennen sind, können (...) nur vorläufig sein“ (Steinvorth 1994: 463).

Die Annahme vielfältiger autonomer Wertsphären und Lebensordnungen scheint nun das Vorhaben der theoriegeleiteten Konstruktion von Brückenhypothesen zu konterkarieren, da die Werte/Güter nicht substituierbar, also auch nicht rational auswählbar sind. Doch der institutionentheoretische Ansatz betrachtet die Autonomie der Wertsphären nicht als Hindernis, sondern als Chance, zu einem 
alternativen Modell der Generierung von Brückenhypothesen zu gelangen. Um dieses Modell zu entwickeln, muss zuerst zwischen institutionellen und instrumentellen Handlungsregeln unterschieden werden. Des Weiteren muss diese Unterscheidung auf das Substitutionsproblem bezogen werden. Die These lautet dann: Instrumentelle Regeln verlangen eine Substitution, während institutionalisierte Regeln eine Substitution verbieten. Kontrastiert man nun Informationen über die Veränderungen in dem Möglichkeitsraum des Handelns mit den Informationen über das Vorliegen/Ausbleiben der Substitution, lässt sich schlussfolgern, ob das vorliegende Verhalten durch instrumentelle oder institutionalisierten Regeln bzw. durch Orientierungen an beiden Regelarten gesteuert wird.

\subsection{Normative und instrumentelle Handlungsregeln}

Als Regeln des Handelns sollen im Folgenden nur praktische Regeln in Abgrenzung zu theoretischen Regeln (oder Regelmäßigkeiten) verstanden werden. Während theoretische Regeln den faktischen Ablauf des Geschehens beschreiben, fordern praktische Regeln (kontrafaktisch) ein bestimmtes Verhalten (Stachura 2008: 10). Praktische Regeln können des Weiteren in drei Kategorien unterteilt werden: in normative, instrumentelle und konstitutive Regeln (ebd.: 10ff.). An dieser Stelle werden die konstitutiven Regeln außer Acht gelassen. Die normativen Regeln unterscheiden sich von den instrumentellen Regeln hinsichtlich ihrer $A n$ wendungsbedingungen und ihrer Geltungsgrundlagen.

\subsubsection{Der Anwendungsaspekt}

Die instrumentellen Regeln nehmen die folgende logische Form an: „Wenn du p willst, dann solltest du q tun "; daher setzt ihre Anwendung einen subjektiven Zweck (d.h. einen vorgestellten, erstrebenswerten Wirklichkeitszustand) voraus. Ohne ein subjektives „Wollen“ wird die instrumentelle Regel also nicht angewandt. Das ist bei den normativen Regeln anders. Diese haben die Form: „du sollst q tun“ oder „wenn die Bedingung $\mathrm{p}$ vorliegt, dann sollst du q tun “ (Elster 1989: 101). Ihre Anwendung kommt also nicht aufgrund eines subjektiv vorgestellten Zwecks, sondern aufgrund einer objektiv vorliegenden situativen Bedingung p zustande. ${ }^{15}$ „Zum Beispiel wird von einer Richterin er-

15 Dies schließt natürlich nicht aus, dass auch bei der Be- wartet, daß sie unpersönlichen (,universalistischen') Standards den Vorrang gibt. Zu Hause, als Mutter, wird von denselben Person erwartet, daß sie persönlichen (,partikularistischen') Standards folgt" (Lindenberg 1990a: 253). Die Anwendung der Regel hängt also nicht von den subjektiven Zwecken und Präferenzen für universalistische oder partikularistische Standards, sondern davon ab, ob sie in den jeweiligen Situationskontext passt. Kontexte wie „Beruf“, „Haushalt“, „Büro“ oder „Partnerschaft" sind selbst institutionelle Konstrukte. Nach M. Rainer Lepsius (1996: 59) kann man hier nach dem Grad fragen, „,in dem der Geltungskontext aus anderen Handlungssituationen ausgegliedert wird“. Dabei gilt: Je ausdifferenzierter die Geltungskontexte, umso präziser können die normativen Handlungsregeln auf spezifische Situationsmuster passen. ${ }^{16}$ In Anschluss an Esser und Kroneberg kann hier zwischen dem Grad der Spezifikation der Situationsmerkmale, der Signifikanz dieser Merkmale für eine Handlungsregel und dem tatsächlichen Vorliegen der Situationsobjekte unterschieden werden (Kroneberg 2007: 218, 226). Alle drei Variablen bestimmen, wie gut eine Handlungsregel in den Kontext passt, sie bestimmen also ihren Passungsgrad (Stachura 2006: 441f.).

Der Unterschied zu den instrumentellen Regeln besteht darin, dass der Anwendungskontext eine Geltungszumutung enthält. Die Regelbefolgung ist nicht „klug“, „nützlich“ oder „notwendig“, son-

folgung von normativen Regeln erstrebenswerte Zweckvorstellungen subjektiv generiert werden. Letztlich ist auch das normative Handeln auf entsprechende Motivationen angewiesen. Aber bei der Orientierung an normativen Regeln kann man sich, im Unterschied zum instrumentellen Handeln, nicht frei entscheiden, ob eine Motivation aufgebaut werden soll oder nicht. Die subjektiv erstrebenswerte Zweckvorstellung ist hier keine Ursache, sondern Folge der Anerkennung der Regelgeltung für eine Situation. Zum Problem der motivationalen Prägung vgl. Albert 2005, 2008.

${ }^{16}$ Es gibt keine „organischen“, „natürlichen“ oder „gesetzmäßigen“ Relationen zwischen wertbezogenen Handlungsregeln und Kontexten. Es ist prinzipiell offen, welche Regeln in welchen Kontexten Geltung erlangen. „Dabei können einzelne Rationalitätskriterien eine Dominanz gegenüber anderen gewinnen und in Lebensbereiche eindringen, aus denen sie ursprünglich nicht erwachsen sind“ (Lepsius 1990: 49). So sind die Regeln der innerweltlichasketischen Lebensführung kein „natürliches“, „universelles" Moment des Kontextes der Wirtschaft, sondern sie wurden in diesen Kontext erst im Zuge der okzidentalen Modernisierung hineingetragen und dort, insbesondere durch den asketischen Protestantismus, erfolgreich installiert. 
dern „richtig“ und „verpflichtend“, „verbindlich“ und „vorbildlich“. Wer sich in einem bestimmten Handlungskontext befindet, der soll sich auch an den dort einschlägigen Regeln orientieren. Ein sicheres Anzeichen des normativen Charakters von Handlungsregeln ist, dass die Akteure nicht subjektiv frei über ihre Geltung verfügen können. Man kann nicht subjektiv frei darüber verfügen, ob z.B. die Personalpolitik in Wirtschaftsunternehmen nach Kriterien der Leistungsfähigkeit oder der erotischen Anziehungskraft, ob wissenschaftliche Publikationen nach den Kriterien der Innovativität und Relevanz oder nach politischer Loyalität bewertet werden sollen. Diesen normativen Charakter der Zuweisung von Regeln oder „Rationalitätskriterien " zu Handlungskontexten meint Lepsius also, wenn er von der Institutionalisierung der Rationalitätskriterien spricht (Lepsius 1990: 44). Unter „Institutionen " können daher normative (sanktionsbewährte) Regeln des Handelns in ausdifferenzierten Geltungskontexten verstanden werden. Im Weiteren wird jedoch undifferenziert von „Institutionen“ oder „normativen Regeln“ gesprochen.

\subsubsection{Der Geltungsaspekt}

Die Geltungsgrundlage der instrumentellen Regeln besteht in den theoretischen Regeln (Regelmäßigkeiten), die einen empirischen Zusammenhang zwischen $\mathrm{q}$ und $\mathrm{p}$ beschreiben („Wenn du q tust, dann wird p passieren“). Normative Regeln entbehren solch einer Grundlage. Es gibt keinen empirischen Zusammenhang zwischen dem Vorliegen einer bestimmten Situation p und einem Verhalten q, auf den sich normative Regeln berufen könnten. Das Rauchverbot bewirkt nicht, dass einem Raucher die Zigarette wie von Geisterhand ausgeht. Das Vorliegen einer Situation $\mathrm{p}$ führt hier empirisch lediglich dazu, dass sich der Akteur im Geltungsbereich einer rechtlichen oder konventionellen Regel befindet. Da normative Regeln nicht auf theoretisch fundiertes Wissen über empirische Regelmäßigkeiten rekurrieren können, müssen sie eine andere Geltungsgrundlage haben. Diese besteht im abgesicherten Wissen über symbolische, im Unterschied zu empirischen Relationen.

Im weberianischen Ansatz sind es Werte, welche die Geltungsgrundlage der normativen Regeln bilden. Vor allem M. Rainer Lepsius nähert sich den normativen Handlungsregeln werttheoretisch an. Das Handeln lässt sich als ein Tun definieren, in dem Werte verwirklicht werden. Aber Wertideen enthalten nach Lepsius keine Hinweise auf die "Methode“ dieses Tuns. Sie sind handlungspraktisch stumm. Man kann an die absolute Verdorben- heit der materiellen Welt und an die daraus abgeleitete Wertidee der „Weltflucht“ glauben. Ob man diese Weltflucht aber durch Gebet, Kontemplation, asketische Übungen oder Erweckung ekstatischer Zustände erreichen soll, ergibt sich aus dem Wertglauben nicht unmittelbar. Es sind normative Handlungsregeln oder Institutionen, die nach Lepsius die "Methode" der Wertverwirklichung bestimmen (Lepsius 1996, 2007). Zwischen Werten und Institutionen besteht eine wechselseitige Relation: Während Institutionen die Werte konkretisieren, stellen Werte die Geltungsgrundlage der Institutionen bereit, d.h., der Geltungsgrad einer Regel bemisst sich daran, wie plausibel diese Regel eine Wertidee zum Ausdruck bringt oder durch diese begründet werden kann. Die Regeln der innerweltlichen Askese sind z.B. eher inadäquate Ausdrucksmittel für den Wert oder das Wertsystem einer weltflüchtigen Religion; die Kontemplationsregeln sind hier viel adäquater, weshalb sie auch in weltflüchtigen Religionen weit höhere Institutionalisierungschancen haben (Obeyesekere 1992: 140ff.).

Die Institutionentheorie von Lepsius setzt an der Schnittstelle zwischen den Werten und Institutionen an, zwischen der „Ebene der symbolischen Deutung der Wertsphären“ und „der Ebene der normativen Geltung“ (Schwinn 2008: 47). Sie fragt danach, welche Wertideen mit welchen Handlungsregeln „verknüpft“ sind und wie „stark“ diese Verknüpfungen ausfallen. Eine wichtige Einsicht des weberianischen Ansatzes besteht darin, dass jede der beiden Ebenen eine Eigendynamik und Eigenlogik aufweist und nicht im Dienste der jeweils anderen Ebene steht. Gleichwohl sind sie aufeinander angewiesen. Daraus entsteht ein Spannungsverhältnis: Obwohl Werte auf die Bereitstellung institutioneller Verwirklichungsmöglichkeiten drängen, vermag die symbolische Ebene die institutionelle nicht zu kontrollieren. Und vice versa: Obwohl die institutionelle Ebene sich gerne durch die Deutungsprodukte der symbolischen legitimieren würde, kann sie den Gang der Deutungsarbeit nicht bestimmen. Die „Verknüpfungen“, die sich zwischen den beiden Ebenen einstellen, können daher unterschiedlich stark ausgeprägt sein, Handlungsregeln können in unterschiedlichen Geltungsgraden mit den Werten verknüpft sein. Wiederum gilt: Je spezialisierter die Arbeit auf der symbolischen Ebene und je ausdifferenzierter die Handlungsregeln, umso größer die Chance für „Verknüpfungen“ mit einem hohem Geltungsgrad. In Analogie zum Passungsgrad einer Regel kann hier von ihrem Geltungsgrad gesprochen werden (Stachura 2006: 441f.). 


\subsection{Lebensordnungen und Produktionsfunktionen}

Nun können die Unterschiede zu Lindenbergs Ansatz explizit gemacht werden: Während die SPFs als instrumentelle Regeln konzipiert werden, stehen normative Regeln im Zentrum des weberianischen Ansatzes. Dieser spricht nicht von „Produktionsfunktionen“, sondern von „Lebensordnungen“, die aus relationierten Werten, normativen Handlungsregeln und Anwendungskontexten bestehen. Dabei bezeichnen die "Geltungsgrade“ der normativen Regeln ihre Relation zu zentralen Werten einer Lebensordnung, während sich der „Passungsgrad“ auf die Relationierung von Regeln und Kontexten des Handelns bezieht.

Der weberianische Ansatz macht sich also für die von der Theorie der SPF vernachlässigten normativsymbolischen Aspekte der Institutionen stark. Doch sein Ziel besteht nicht darin, eine materialistische durch eine normative Vereinseitigung zu ersetzen. Die Lebensordnungen sind zwar primär normative Gebilde, aber sie umfassen auch instrumentelle Handlungsregeln, die der Herstellung von Zwischen- und Nebenprodukten der Lebensordnungen dienen. Solche Nebenprodukte sind soziale Güter wie z.B. Macht, Prestige, soziale Anerkennung oder materielle Belohnung für das Handeln in institutionalisierten Lebensordnungen. In jeder Lebensordnung können solche Güter, die oft nicht ordnungsspezifisch, sondern ordnungsübergreifend einsetzbar sind, zu Motiven des Handelns werden ${ }^{17}$ Bei der Konstruktion von Brückenhypothesen müssen beide Regelarten berücksichtigt werden.

\subsection{Die Regelarten und zwei Richtungen der Handlungsanpassung}

Normative und instrumentelle Regeln haben entgegengesetzte Eigenschaften. Während die Geltung Ersterer durch Werte garantiert wird, leitet sich die Geltung der Letzterer von ihrer Wirksamkeit hinsichtlich der Herstellung von Nebenprodukten einer Lebensordnung ab. Während die Anwendung jener durch situative Kontexte gesteuert wird, werden diese durch subjektive Zielvorstellungen aktiviert. Daraus folgt: Während normative Regeln die

\footnotetext{
17 Wie der Neoinstitutionalismus zeigt, können die als geltend anerkannten Kriterien das tatsächliche Handeln oft nicht erklären. In einer berühmten Studie über Rekrutierungsverfahren an einer amerikanischen Universität haben March und Olsen die geringere Erklärungskraft der raumspezifischen Bewertungskriterien demonstriert (March/Olsen 1987: 114).
}

Substitution verbieten, fordern instrumentelle Regeln ein Substitutionsverhalten.

Der Grundgedanke des hier entwickelten Ansatzes kommt am deutlichsten vor dem Hintergrund der Kernannahme des ökonomischen Ansatzes zum Vorschein: Die Befolgung von Handlungsregeln erzeugt generell Kosten. Die relativen Kosten können steigen, wenn die Mittel des Handelns teurer werden oder wenn das Einkommen der Akteure sinkt. Der ökonomische Ansatz geht davon aus, dass es bei einer Veränderung von Restriktionen und Opportunitäten zu einer Anpassung der Produktion an den neuen Restriktionsraum des Handelns kommt; wird ein Produktionsfaktor teurer, wird weniger dieses Faktors eingesetzt. Aus der Perspektive des weberianischen Ansatzes ist das nur eine theoretische Möglichkeit. Eine andere Möglichkeit besteht darin, dass bei der Verteuerung eines „Produktionsfaktors" nicht weniger von diesem „Faktor" eingesetzt, sondern mehr für die als „richtig “ angesehene Menge bezahlt wird. In dem einen Falle passt sich das Handeln dem Restriktionsraum an. In dem anderen Falle passt man das Handeln dem normativen Ideal an, indem man die normativ geforderte Handlung unabhängig von den Kosten, Preisen und Opportunitäten vollzieht. Man könnte auch von zwei Anpassungsrichtungen des Handelns sprechen. ${ }^{18}$ Der ökonomische Ansatz kennt demgegenüber nur die eine Richtung: „Streng rationales Handeln, - so kann man es auch ausdrücken, - wäre glatte und restlose ,Anpassung' an die gegebene ,Situation'. Die Mengerschen theoretischen Schemata z.B. enthalten die streng rationale ,Anpassung' an die ,Marktlage" als Voraussetzung in sich " (Weber 1988: 227; vgl. Schluchter 2005: 25, Fn. 47).

Nun kann man die unterschiedlichen Richtungen der Anpassung mit der Unterscheidung zwischen normativen und instrumentellen Handlungsregeln in Zusammenhang bringen. Während bei der Orientierung an instrumentellen Handlungsregeln eine Anpassung an den Möglichkeitsraums des Handelns impliziert wird, fordern Institutionen eine Anpassung an normative Ideale. Der weberianische Ansatz betont also die theoretische Relevanz beider Anpassungsrichtungen, und er kann auch bestimmen, wann die eine und wann die andere Richtung $\mathrm{zu}$ erwarten ist: Bei einer Handlungsregel, deren Geltung aus einer anerkannten Beziehung auf eine Wertidee resultiert und die einen hohen Passungsgrad auf einen real gegebenen Kontext aufweist, ist nicht von einer Anpassung an die "Marktlage“,

${ }^{18}$ Die Unterscheidung von Anpassungsrichtungen des Handelns geht bekanntlich auf Searle (1982: 19) zurück. 
sondern von einer umgekehrten Anpassung an das normativ geforderte Ideal gegen die Marktlage auszugehen. Das heißt, die Verteuerung von „Produktionsfaktoren" wird hier prinzipiell keine Einschränkung des Handelns nach sich ziehen (Gleiches gilt für eine Preissenkung). Daher können z.B. weder massive Repressionen (Preise) noch eine massive Wohlstandssteigerung (Opportunitätskosten) die Intensität religiösen Handelns beeinflussen, wenn die religiöse Lebensordnung ihre Geltung und Anwendungskontexte behält. Die Resistenz des Handelns gegen Verlockungen und Substitutionsanreize zeigt sich auch an profaneren Handlungsarten. Extremsportler, die mitunter erhebliche gesundheitliche Nebenfolgen in Kauf nehmen, oder Kurzstreckenfahrer, die im Namen eines normativen Ideals der motorisierten Fortbewegung lange Zeiten im Stau stehen, richten sich auch nicht nach einer, sondern gegen eine „Marktlage“. Bei einem schwachen Geltungs- und Passungsgrad können hingegen schon geringe Änderungen von Restriktionen und Opportunitäten Substitutionsverhalten auslösen. Die theoretische Unterscheidung zwischen beiden Anpassungsrichtungen macht deutlich, wie in der sozialen Wirklichkeit hohe Nutzenresistenz und fieberhafte Nutzenmaximierung nebeneinander fortbestehen können.

\subsection{Brückenhypothesen}

Wie lässt sich nun die Unterscheidung zwischen den beiden Anpassungsrichtungen für die Generierung von Brückenhypothesen nutzen? Jedes Handeln kann sowohl an normativen als auch an instrumentellen Regeln orientiert sein. Wie kann man nun wissen, auf welche Regelorientierung eine Handlung zurückgeht?

Zwar können wir über die institutionellen Geltungskontexte bestimmte Regeln identifizieren, die eine Handlung erklären könnten, doch auf diese Weise lassen sich meist mehrere Regeln auslesen. Wie kann die richtige identifiziert werden? Die Grundidee dazu lässt sich wie folgt beschreiben: Um herauszufinden, welche Handlungsregel eine Handlung geleitet hat, muss man jene Regeln, die diese Handlung potenziell erklären können, mit den Informationen über den Restriktionsraum des Handelns kontrastieren. Der Handlungsverlauf kann sich dabei entweder gleich- oder gegenläufig zur Entwicklung des Restriktionsraums verhalten. Nehmen wir an, dass die zu erklärende Handlung in der Intensivierung religiösen Handelns in einer Gruppe von Menschen besteht. Einfachheitshalber konstruieren wir nur zwei Regeln, die das Explanandum erklären können. Nach der einen Regel würde die Einhaltung religiöser Gebote Wohlstand für die Gruppe bringen $\left(\mathrm{R}_{1}\right)$. Nach der anderen Regel würde die Einhaltung der Gebote von einem transzendenten Wesen gefordert sein $\left(\mathrm{R}_{2}\right)$. Um zu bestimmen, welche der beiden Regeln die Handlung geleitet hat, muss man sich den Restriktionsraum des Handelns anschauen. Stellt sich dabei heraus, dass sich das religiöse Handeln insgesamt verteuerte, etwa indem die Mitglieder der religiösen Gruppe Repressionen unterworfen wurden oder sonstige Opportunitätskosten gestiegen waren, dann hat man es mit gegenläufigen Tendenzen bei der Handlungsentwicklung und der Entwicklung im Restriktionsraum zu tun. Gemäß der Unterscheidung zwischen den beiden Anpassungsrichtungen des Handelns ist $\mathrm{zu}$ vermuten, dass gegenläufige Tendenzen nicht von instrumentellen, sondern nur von normativen Handlungsregeln erklärt werden können. Man muss also prüfen, um welche Regeln es sich bei $R_{1}$ und $R_{2}$ handelt. Ist die $R_{1}$ instrumentell, dann scheidet sie als Erklärungsregel des fraglichen Handelns aus. Handelt es sich bei $R_{2}$ ebenfalls um eine instrumentelle Regel, kann das Handeln auf der gegebenen Grundlage nicht erklärt werden; weitere Regeln müssen herangezogen werden. Ergibt sich aus der Analyse des logischen Gehalts der $\mathrm{R}_{2}$, dass es sich dabei um eine institutionalisierte Regel handelt, dann müssen folgende Merkmale geprüft werden: 1. ihr Geltungsgrad, 2. die Art der Kontexte, die sie normativ (mit einem bestimmten Nachdruck) verlangt, und 3. das tatsächliche Vorliegen dieser Kontexte in der Situation, in der gehandelt wurde. Ergibt sich bei der Rekonstruktion der Geltungskonzeptionen der religiösen Lebensordnung, dass $\mathrm{R}_{2}$ einen hohen Geltungsgrad in Bezug auf die zentrale Wertidee der Ordnung aufweist, einen bestimmten Kontext mit einem bestimmten Nachdruck verlangt und der Kontext auch tatsächlich vorliegt, dann kann man davon ausgehen, dass $\mathrm{R}_{2}$ das Handeln auch erklären kann (vgl. den Fall des kostenresistenten Handelns in Abb. 1).

Gegenläufige Tendenzen können aber auch im umgekehrten Fall dazu führen, dass eine Handlungsweise nicht intensiviert, sondern trotz günstiger Opportunitäten reduziert oder aufgegeben wird. Ein Beispiel stellt die Deinstitutionalisierung der Sklaverei in den USA zu einem Zeitpunkt dar, in dem diese Institution „offensichtlich noch einträglich" war (North 1992: 100). Das Handeln ist hier „durch die Veränderung der relativen Preise nicht völlig zu erklären“, da die Opportunitäten nicht 


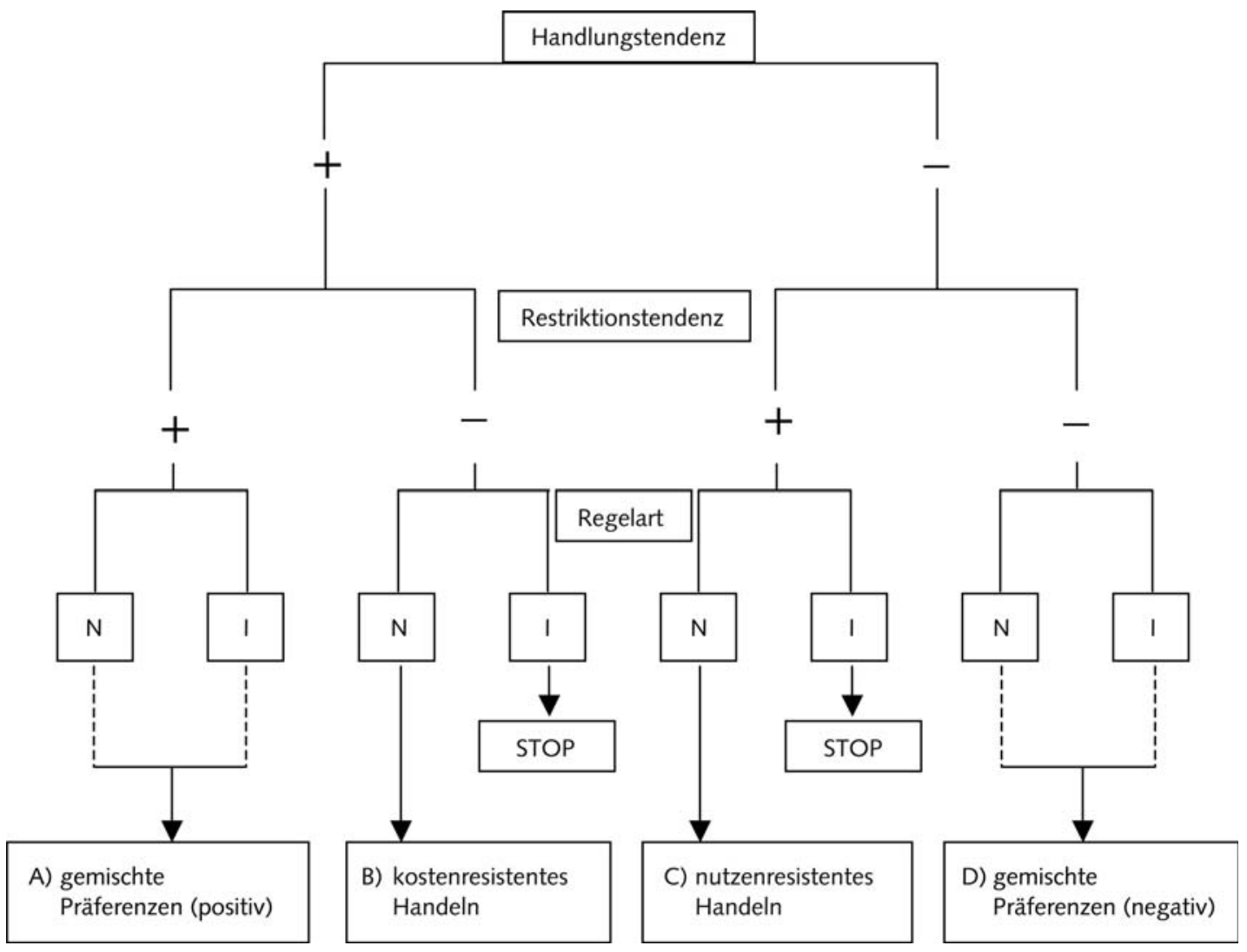

Abb. 1 Konstellationsanalytische Konstruktion von Brückenhypothesen $\left({ }^{+}+{ }^{\prime \prime}=\right.$ positive Tendenz; - $^{\text {" }}=$ negative Tendenz; "N“ = normativ-institutionelle Regeln, "I" = instrumentelle Regeln)

für, sondern gegen die Einschränkung der Produktion sprechen (ebd.). Wiederum erbringt der weberianische Ansatz hier einen explanativen Mehrwert gegenüber dem Modell der SPF. Während dieses die Veränderung im Ablauf des Handelns nur mit der Verknappung (bzw. Vermehrung) von Ressourcen erklären kann, wird die institutionentheoretische Alternative auch das Verschwinden von Handlungsregeln oder Geltungskontexten als mögliche Ursachen mit in Betracht ziehen. Lassen sich nun institutionalisierte Handlungsregeln rekonstruieren, die einen hohen Geltungsgrad in Bezug auf bestimmte Wertideen aufweisen, die spezifische Geltungskontexte verlangen, und können in der Wirklichkeit des Handelns diese Kontexte ausfindig gemacht werden, dann kommen diese Regeln als gute Kandidaten für die Erklärung nutzenresistenten Verhaltens in Betracht (vgl. den Fall des nutzenresistenten Handelns in Abb. 1).

Generell kann man sagen, dass gegenläufige Tendenzen eine Orientierung an normativen Regeln an- zeigen, während gleichläufige Tendenzen eine Orientierung an instrumentellen Regeln signalisieren. Aber das Verhältnis ist nicht ganz symmetrisch, denn während gegenläufige Tendenzen die instrumentellen Regeln ausschließen, werden institutionalisierte Regeln durch gleichläufige Tendenzen nicht notwendigerweise ausgeschlossen. Es kann nämlich auch gemischte Orientierungen geben, d.h. solche, die sowohl normative als auch instrumentelle Regeln enthalten. Gemischte Präferenzen sind für die Konstruktion von Brückenhypothesen grundsätzlich nicht problematisch. Problematisch ist nur ihre Unterstellung, und zwar dann, wenn erstens, die Regeln entgegengesetzte Anpassungsrichtungen verlangen oder zweitens die Regeln untereinander im Konflikt stehen. Die Wirtschaftsethik des asketischen Protestantismus hat z.B. den „,Erwerbstrieb“ aller jener Gründer und Spekulanten, die ,mit dem Ärmel an das Zuchthaus streifen', um die Millionen zu verdienen“ (Weber 1910: 195), verpönt. Der Grund, weshalb der „Erwerbstrieb“ keine Ursache 
der protestantischen Lebensführung war, liegt hier in dem institutionellen Konflikt zwischen der reinen Gewinnorientierung und wirtschaftsethischen Handlungsregeln. Man kann nicht zugleich glauben, dass man das religiöse Ziel (Erlösungsgewissheit) nur dann erreicht, wenn man den Erwerbstrieb ausschaltet, und erwerbstriebgesteuert handeln (wollen). Eine Brückenhypothese, die wirtschaftsethische und gewinnorientierte Bewertungskriterien als Grundlage der Handlung annehmen würde, wäre trotz gleichläufiger Tendenz falsch. Man darf die „Nutzenargumente“ nicht beliebig mischen und kombinieren. Solch ein instrumentalistischer Fehlschluss liegt natürlich nicht vor, wenn keine institutionellen Konflikte erkennbar sind. Gemischte Präferenzen können also bei gleichläufigen Tendenzen und unter Abwesenheit institutioneller Konflikte angenommen werden. Sie können ferner nicht nur bei einer positiven (vgl. den Fall $A$ in Abb. 1), sondern auch bei einer negativen Gleichläufigkeit der Handlung und der Restriktionen bedeutsam für die Handlungserklärung sein (vgl. den Fall $D$ in Abb. 1). Sowohl der Zerfall des Ordnungshandelns als auch dessen Aufrechterhaltung kann von gemischten Orientierungen getragen werden.

\section{Ausblick: Eine theoriegesteuerte Konstruktion von Brückenhypothesen?}

Ausgangspunkt der vorliegenden Überlegungen war das Dilemma zwischen der empirischen und der theoriegesteuerten Konstruktion von Brückenhypothesen für die verstehende Erklärung sozialen Handelns, d.h. einer Erklärung, die auf dem Prinzip „Gründe als Ursachen“ des Handelns basiert. Zugespitzt formuliert, hat die empirische Vorgehensweise ein Problem mit dem erklärenden Aspekt des Modells, da sie den Zusammenhang zwischen den abgefragten Präferenzen und den intersubjektiv geltenden Handlungsregeln der Akteure leicht aus den Augen verlieren kann. Des Weiteren hat sie ein Problem mit der Selektion der kausal relevanten Präferenzen der Akteure. Bei der theoriegeleiteten Vorgehensweise wird die Verstehen-Komponente vernachlässigt. Der Theoretiker legt großes Gewicht auf die Selektion der kausal relevanten Präferenzen; die von ihm konstruierten Selektionsregeln kommen aber in der Lebenswelt der agierenden Akteure nicht vor.

Die kritische Analyse des Modells der theoriegesteuerten Konstruktion von Brückenhypothesen von Siegwart Lindenberg hat gezeigt, dass es aus diesem Dilemma kein Entrinnen gibt. Gleichwohl hat Lindenberg einen Weg vorgezeichnet, auf dem zwar keine strikte Lösung, aber immerhin eine Abschwächung des Problems zu erreichen ist. Es handelt sich dabei um eine institutionenanalytische Vorgehensweise. Das Wissen über geltende Institutionen ist unabhängig von subjektiven Befindlichkeiten und momentanen Erinnerungen der Akteure. Es lässt sich daher auch relativ zuverlässig erheben. Auf diese Weise kann Lindenberg plausibel machen, wie die „Produktionsfunktionen“, die im Wesentlichen aus Institutionen bestehen, empirisch rekonstruiert werden können.

Unplausibel ist aber die Annahme, dass alle „Produktionsfunktionen “ ihrerseits nur Argumente einer übergreifenden Endnutzen-Produktionsfunktion darstellen, und zwar deshalb, weil solch eine Funktion keine institutionelle Entsprechung oder Deckung mehr hat. Die Vorstellung einer institutionellen Dachkonstruktion, die alle gesellschaftlichen „Teilbereiche“, „Systeme“ oder „Lebensordnungen“ umspannen würde, ist vielmehr ausgesprochen vormodern. Die Endnutzen-Produktionsfunktion hat bei Lindenberg selbst aber nur die Funktion, das nomologisch-theoretische Modell der Konstruktion von Brückenhypothesen zu ermöglichen. Die hier entwickelte Alternative räumt mit der Vorstellung des „Endnutzens“ auf. Ist sie deshalb theorielos? Das ist sie nicht, denn auf der Grundlage der Unterscheidung zwischen institutionellen und instrumentellen Regeln entwickelt sie ein theoretisches Ausschlussverfahren für die Bestimmung der erklärungsfähigen Handlungsregeln. 2

Durch die Einsicht in die Vielfalt irreduzierbarer Wertsphären vermeidet das Modell eine materialistische Verengung; die Einsicht in die strukturelle Einbettung des Handelns verhindert wiederum eine idealistische Verengung. Vor allem aber zeichnet der institutionentheoretische Ansatz das Bild eines bindungsfähigen Akteurs, der in einer Welt vielfältiger Werte lebt und diese Welt auch zu reproduzieren imstande ist.

\section{Literatur}

Albert, G., 2005: Moderater methodologischer Holismus. Eine weberianische Interpretation des Makro-MikroMakro-Modells. Kölner Zeitschrift für Soziologie und Sozialpsychologie 57: 387-413.

Albert, G., 2007: Keines für alle! Kölner Zeitschrift für Soziologie und Sozialpsychologie 59: 340-349.

Albert, G., 2008: Soziologie mittlerer Reichweite. S. 445467 in: S. Sigmund / G. Albert / A. Bienfait / M. Stachura (Hrsg.), Soziale Konstellation und historische Perspektive. Wiesbaden: VS. 
Bourdieu, P., 2006: Die feinen Unterschiede. Kritik der gesellschaftlichen Urteilskraft, Suhrkamp-Taschenbuch Wissenschaft. Frankfurt a.M.: Suhrkamp.

Coleman, J.S., 1991: Grundlagen der Sozialtheorie, Bd. 1. München: Oldenbourg.

Eckhard, J., 2008: Partnerschaftswandel und Geburtenrückgang: Eine Analyse des westdeutschen Geburtenrückgangs im Kontext veränderter Partnerschaftsverläufe. Heidelberg: unv. Manuskript.

Elster, J., 1989: The Cement of Society: A Study of Social Order. Cambridge: Cambridge University Press.

Esser, H., 1993: Soziologie. Allgemeine Grundlagen. Frankfurt a.M.: Campus.

Esser, H., 2000a: Soziologie: Spezielle Grundlagen. Band 1: Situationslogik und Handeln. Frankfurt a.M.: Campus.

Esser, H., 2000b: Soziologie: Spezielle Grundlagen. Band 4. Opportunitäten und Restriktionen. Frankfurt a.M.: Campus

Etzrodt, C., 2007: Neuere Entwicklungen in der Handlungstheorie. Zeitschrift für Soziologie, 36: 364-379.

Greshoff, R., 2006: Das Essersche „Modell der soziologischen Erklärung “ als zentrales Integrationskonzept im Spiegel der Esser-Luhmann-Weber-Vergleiche - was resultiert für die weitere Theoriediskussion? S. 515-580 in: R. Greshoff / U. Schimank (Hrsg.), Integrative Sozialtheorie? Esser - Luhmann - Weber. Wiesbaden: VS.

Greve, J. / Schnabel, A. / Schützeichel, R. (Hrsg.), 2008: Das Mikro-Makro-Modell der soziologischen Erklärung. Zur Ontologie, Methodologie und Metatheorie eines Forschungsprogramms. Wiesbaden: VS.

Kelle, U., 1994: Empirisch begründete Theoriebildung, zur Logik und Methodologie interpretativer Sozialforschung. Weinheim: Dt. Studien-Verl.

Kelle, U. / Lüdemann, C., 1995: „Grau, teurer Freund, ist alle Theorie..." Rational Choice und das Problem der Brückenannahmen. Kölner Zeitschrift für Soziologie und Sozialpsychologie 47: 249-267.

Kroneberg, C., 2005: Die Definition der Situation und die variable Rationalität der Akteure. Zeitschrift für Soziologie 34: 344-363.

Kroneberg, C., 2007: Wertrationalität und das Modell der Frame-Selektion. Kölner Zeitschrift für Soziologie und Sozialpsychologie 59: 215-240.

Lepsius, M.R., 1990: Interessen, Ideen und Institutionen. Opladen: Westdeutscher Verlag.

Lepsius, M.R., 1996: Institutionalisierung und Deinstitutionalisierung von Rationalitätskriterien. S. 57-69 in: G. Göhler (Hrsg.), Institutionenwandel. Leviathan, Sonderheft 16. Opladen. Westdeutscher Verlag.

Lepsius, M.R., 2007: Max Weber. Begründer der modernen Sozialwissenschaften. in: U. Leutheusser / H. Nöth (Hrsg.), München leuchtet für die Wissenschaft S. München: Allitera Verlag.

Lindenberg, S., 1990a: Rationalität und Kultur. S. 249287 in: H. Haferkamp (Hrsg.), Sozialstruktur und Kultur. Frankfurt a.M.: Suhrkamp.

Lindenberg, S., 1990b: Model of Man in the Social Sciences. Journal of Institutional and Theoretical Economics 146: 727-748.

Lindenberg, S., 1992: An Extended Theory of Institutions and Contractual Discipline. Journal of Institutional and Theoretical Economics 148: 125-154.

Lindenberg, S., 1996a: Theoriegesteuerte Konkretisierung der Nutzentheorie. Kölner Zeitschrift für Soziologie und Sozialpsychologie 48: 560-565.

Lindenberg, S., 1996b: Die Relevanz theoriereicher Brückenannahmen. Kölner Zeitschrift für Soziologie und Sozialpsychologie 48: 126-140.

Lüdemann, C. / Rothgang, H., 1996: Eine Kritik der Framing-Modelle von Siegwart Lindenberg und Hartmut Esser. Zeitschrift für Soziologie 25: 278-288.

March, J.G. / Olsen, J.P., 1987: Ambiguity and Choice in Organizations Bergen: Universitetsforlaget.

Nauck, B., 2001: Der Wert von Kindern für ihre Eltern. Kölner Zeitschrift für Soziologie und Sozialpsychologie 53: 407-435.

North, D.C., 1988: Theorie des institutionellen Wandels. Tübingen: Mohr Siebeck.

North, D.C., 1992: Institutionen, institutioneller Wandel und Wirtschaftsleistung Tübingen: Mohr Siebeck.

Obeyesekere, G., 1992: Buddhismus: Die Begegnung einer Achsenzeitreligion mit dem Modernismus. S. 101-150 in: S.N. Eisenstadt (Hrsg.), Kulturen der Achsenzeit II. Ihre institutionelle und kulturelle Dynamik Teil 3. Frankfurt a.M.: Suhrkamp.

Opp, K.-D. / Friedrichs, J., 1996: Brückenannahmen, Produktionsfunktionen und die Messung von Präferenzen. Kölner Zeitschrift für Soziologie und Sozialpsychologie 48: 546-559.

Putnam, R.D. / Leonardi, R. / Nanetti, R.Y., 1994: Making Democracy Work: Civic Traditions in Modern Italy. Princeton: Princeton University Press.

Riesebrodt, M., 2000: Die Rückkehr der Religionen: Fundamentalismus und der „Kampf der Kulturen“. München: Beck.

Rohwer, G., 2003: Modelle ohne Akteure: Hartmut Essers Erklärung von Scheidungen. Kölner Zeitschrift für Soziologie und Sozialpsychologie 55: 340-358.

Schluchter, W., 2005: Handlung, Ordnung und Kultur: Studien zu einem Forschungsprogramm im Anschluss an Max Weber. Tübingen: Mohr Siebeck.

Schmid, M., 2004: Rationales Handeln und soziale Prozesse. Wiesbaden: VS.

Schwinn, T., 2001: Differenzierung ohne Gesellschaft: Umstellung eines soziologischen Konzepts. Göttingen: Velbrück.

Schwinn, T., 2006: Der Nutzen der Akteure und die Werte der Systeme. S. 39-62 in: R. Greshoff / U. Schimank (Hrsg.), Weber und Esser: Gesellschaft, Sinn und Handeln. Wiesbaden: VS.

Schwinn, T., 2008: Institutionenanalyse und Makrosoziologie. S. 43-69 in: M. Stachura / A. Bienfait / G. Albert / S. Sigmund (Hrsg.), Der Sinn der Institutionen. Eine Mehr-Ebenen und Mehr-Seiten-Analyse. Wiesbaden: VS.

Searle, J.R., 1982: Ausdruck und Bedeutung. Untersuchungen zur Sprechakttheorie Frankfurt a.M.: Suhrkamp.

Srubar, I., 1992: Grenzen des „Rational-Choice“-Ansatzes. Zeitschrift für Soziologie 21: 157-165. 
Stachura, M., 2006: Logik der Situationsdefinition und Logik der Handlungsselektion. Der Fall des wertrationalen Handelns. Kölner Zeitschrift für Soziologie und Sozialpsychologie 58: 433-452.

Stachura, M., 2008: Einleitung. Der Standort weberianischer Institutionentheorie im Raum konkurrierender Forschungsprogramme. S. 8-39 in: M. Stachura / A. Bienfait / G. Albert / S. Sigmund (Hrsg.), Der Sinn der Institutionen. Eine Mehr-Ebenen- und Mehr-SeitenAnalyse. Wiesbaden: VS.

Steinvorth, U., 1994: Webers Freiheit von der Wertfreiheit. S. 445-472 in: G. Wagner, / H. Zipprian (Hrsg.),
Max Webers Wissenschaftslehre. Frankfurt a.M.: Suhrkamp.

Stigler, G.J. / Becker, G.S., 1977: De Gustibus Non Est Disputandum. American Economic Review 67: 76-90.

Weber, M., 1910: Antikritisches zum „Geist“ des Kapitalismus. Archiv für Sozialwissenschaft und Sozialpolitik 30: 176-202.

Weber, M., 1976: Wirtschaft und Gesellschaft: Grundriss der verstehenden Soziologie. Tübingen: Mohr Siebeck

Weber, M., 1988: Gesammelte Aufsätze zur Wissenschaftslehre. Tübingen: Mohr Siebeck.

\section{Autorenvorstellung}

Mateusz Stachura, geb. 1975 in Katowice. Studium der Politischen Wissenschaft und Soziologie in Krakau und Heidelberg. Promotion in Heidelberg. Seit 2003 wissenschaftlicher Mitarbeiter an der Universität Heidelberg.

Forschungsgebiete: Handlungstheorie, Institutionentheorie, individualistisch-strukturalistischer Ansatz, politische Kultur-Forschung.

Veröffentlichungen: Deutung des Politischen. Ein handlungstheoretisches Konzept der politischen Kultur und seine Anwendung. Frankfurt 2005; Logik der Situationsdefinition und Logik der Handlungsselektion. Der Fall des wertrationalen Handelns. KZfSS 58, 2006: 433-452; Einleitung: Der Standort weberianischer Institutionentheorie im Raum konkurrierender Forschungsprogramme, in: M. Stachura / A. Bienfait / G. Albert / S. Sigmund (Hrsg.), Der Sinn der Institutionen. Eine Mehr-Ebenen- und Mehr-Seiten-Analyse. Wiesbaden 2008. 